\title{
Contribution à l'étude de la fraction insaponifiable de trois huiles d'olive issues des variétés Guasto, Rougette et Blanquette plantés dans l'est algérien
}

\author{
Monia MEZGHACHE ${ }^{1}$ \\ Cherifa HENCHIRI ${ }^{1}$ \\ Lucy MARTINE ${ }^{2}$ \\ Olivier BERDEAUX ${ }^{2}$ \\ Noureddine AOUF \\ Pierre JUANEDA ${ }^{2}$ \\ ${ }^{1}$ Laboratoire de biochimie appliquée, \\ Université Badji Mokhtar, \\ Annaba-Algerie \\ ${ }^{2}$ Centre des sciences du goût et de l'alimentation, \\ UMR6265 CNRS, UMR1324 INRA, \\ Université de Bourgogne, \\ Agrosup, Dijon, \\ France \\ ${ }^{3}$ Laboratoire de chimie organique appliquée \\ (LCOA), \\ Université Badji Mokhtar, \\ Annaba-Algérie
}

Article reçu le 8 juillet 2009

accepté le 6 juillet 2010

\begin{abstract}
The sterol profile of three Algerian virgin olive oils produced from Guasto, Blanquette and Rougette cultivar's, varieties grown in the East of the country, was established by gas chromatography using a flame ionization detector and coupled to mass spectrometry. Four sterol compounds were identified and characterized in the three oils which are, sitosterol always predominant (43-66\%), delta 5-avenastérol (8-13\%), stigmasterol ( $\leq 1,5 \%)$ and campesterol (1-2\%); one methyl sterol (4-methylsterol) : citrostadienol (4-7\%) and two triterpenic alcohols: 24-methylene cycloartanol (10-33\%) and cycloartenol (3-13\%). These results vary according to campany and variety. Our study shows that most of these compounds were affected by climatic conditions especially during the campanies 2004-2005 and 2005-2006. The amounts of the four identified sterols are in agreement with the European regulation (Reg. (EEC) $n^{\circ} 2568 / 91$, appendix 1).
\end{abstract}

Key words: phytosterols, olive oil, cultivars, Olea europaea

\section{Introduction}

L'huile d'olive est une des principales composantes du régime dit « méditerranéen », connu pour son action bénéfique sur la santé (Jacotot, 1996). Elle est caractérisée par sa composition particulière en acides gras, en composés mineurs appartenant à la fraction insaponifiable des huiles végétales. Ses caractéristiques physicochimiques et organoleptiques sont définies par la norme commerciale du Conseil Oléicole International (COI, 2009) et au niveau européen, par l'annexe I du règlement (CEE) $n^{\circ}$ 2568/91 (CEE, 1991).

Elle contient une forte teneur en acide gras mono-insaturés représentée par l'acide oléique de la famille oméga-9 (65 à $80 \%$ ), $15 \%$ d'acides gras saturés et $10 \%$ d'acides gras polyinsaturés représentés par l'acide linoléique - oméga- 6 dominant et des traces de l'acide $\alpha$-linolénique oméga-3, acides gras essentiels, indispensables car non synthétisables par l'organisme humain.

Les composés mineurs sont représentés par les phénols, tocophérols, caroténoïdes et les stérols, objet de cette étude. Plusieurs paramètres influencent la composition stérolique de I'huile comme par exemple, la variété et le degré de maturité des fruits (Hajana et al., 1998) les méthodes d'extraction (Di Giovaccino, 1996), de conservation de I'huile (Gutierrez et al., 2000) et enfin les conditions climatiques et agronomiques (El Antari et al., 2000).

Les plus importants des stérols sont le sitostérol, le campestérol et le stigmastérol, qui sont habituellement consommés en faibles quantités dans un régime alimentaire traditionnel n'apportant pas plus de $300 \mathrm{mg}$ de phytostérols/jour.

Récemment, des études ont proposé que le profil stérolique puisse être utilisé pour classer les huiles d'olives vierge en fonction de leur variété (Bucci et al., 2002 ; Ranalli et al., 2002 ; El Antari et al., 2000). C'est aussi un paramètre très important pour contrôler la pureté des huiles d'olive et y détecter des adultérations par la présence d'huiles de différentes origines (Ollivier, 2003).

L'absence de données sur les caractéristiques des huiles d'olive algériennes nous a conduits à entreprendre ce travail. Celui-ci a pour objectif de caractériser ces huiles d'olive par leurs compositions en stérols et autres constituants de l'insaponifiable en fonction du lieu géographique, de la nature du sol, de la région de culture et des cultivars. Ainsi, nous avons étudié la variation de la composition, notamment stérolique, de trois huiles d'olive issues de trois cultivars dominants à l'est algérien (wilaya de Skikda) à savoir la Guasto, la Blanquette et la Rougette pendant trois campagnes consécutives 2004-2005, 2005-2006 et 2006-2007. 


\section{Matériels et méthodes}

\section{Matériel végétal}

Trois cultivars dominants, plantés dans deux régions de l'est algérien (Es-Sebt et Oued el Aneb : wilaya de Skikda) à savoir Guasto, Blanquette et Rougette ont fait I'objet de cette étude. La figure 1 et le tableau 1 présentent respectivement les aires géographiques de production et la caractérisation primaire des trois cultivars étudiés. Les échantillons ont été récoltés au mois de décembre pendant trois campagnes successives 2004-2005, 2005-2006 et 2006-2007.

Les deux régions sont caractérisées par des reliefs montagneux avec des sols peu évolués (région Es-Sebt), brunifiés et contenant des sesquioxydes de fer d'apport alluviaux récents (sols profonds généralement de texture limoneuse ou argileuse) (Oued el Aneb) (Itaf, 2002).

La wilaya de Skikda appartient à l'étage bioclimatique subhumide supérieur sous influence maritime. Le climat est de type méditerranéen avec des températures moyennes annuelles minimales entre 11 et $14{ }^{\circ} \mathrm{C}$ et des températures maximales entre 21 et $25^{\circ} \mathrm{C}$ (pouvant atteindre 44 à $49{ }^{\circ} \mathrm{C}$ ). La pluviométrie est supérieure en moyenne à $650 \mathrm{~mm} / \mathrm{an}$. Les précipitations sont plus abondantes sur les sommets que sur les plaines et les vallées (Anonyme, 2007).

En ce qui concerne les trois campagnes :

- Campagne 2004-2005: année avec une pluviométrie normale (précipitations assez bonnes) et des températures estivales élevées avec forte tendance à la sècheresse (Anonyme, 2005).

- Campagne 2005-2006 : peu de pluviométrie, tendance à la sècheresse (Anonyme, 2006).

- Campagne 2006-2007: bonne année agricole (température et pluviométrie), meilleure que les deux précédentes (Anonyme, 2007).

\section{Extraction de l'huile}

L'extraction a été réalisée dans une huilerie par procédé en continu selon les étapes suivantes: lavage, broyage, pétrissage et centrifugation (figure 2). C'est un procédé italien Alfa Laval qui fonctionne avec un décanteur par centrifugation à deux phases (huile et grignons) qui ne demande pas d'eau pour la séparation de l'huile (liquide) des grignons et margines (solides). Avec ce procédé, le rendement est plus élevé et I'huile obtenue, de meilleure qualité est plus riche en composés phénoliques que celle obtenue avec le procédé ancien à trois phases (Anonymea, 2006). Les huiles ont été prélevées dès la première pression. Les échantillons récupérés ont été conservés au réfrigérateur à une température de $+4{ }^{\circ} \mathrm{C}$ en attendant d'être analysés.

\section{Dosage des stérols et autres constituants de l'insaponifiable}

L'analyse de ces composés nécessite trois étapes successives :

\section{Saponification à froid des lipides totaux}

Cette première étape à pour but d'isoler la fraction insaponifiable des lipides totaux. $20 \mathrm{~mL}$ de potasse à $1 \mathrm{M}$ dans le méthanol (Merck 5012) ont été ajouté à $500 \mathrm{mg}$ d'huile. Après une agitation énergique, les échantillons ont été placés sous argon dans le noir pendant toute une nuit. Cette méthode a été choisie pour sa douceur et permet d'éviter la formation d'oxystérols lors de la manipulation (Grandgirard et al., 2004).

L'échantillon saponifié est versé dans une ampoule à décanter de $100 \mathrm{~mL}$, puis extrait avec $40 \mathrm{~mL}$ d'eau osmosée et $40 \mathrm{~mL}$ de dichlorométhane (SDS ref 2950A48). Après décantation de l'échantillon, la phase inférieure est recueillie dans un ballon de $100 \mathrm{~mL}$.

La phase aqueuse est extraite une deuxième fois avec $40 \mathrm{~mL}$ de dichlorométhane, la phase inférieure organique est récupérée dans le même

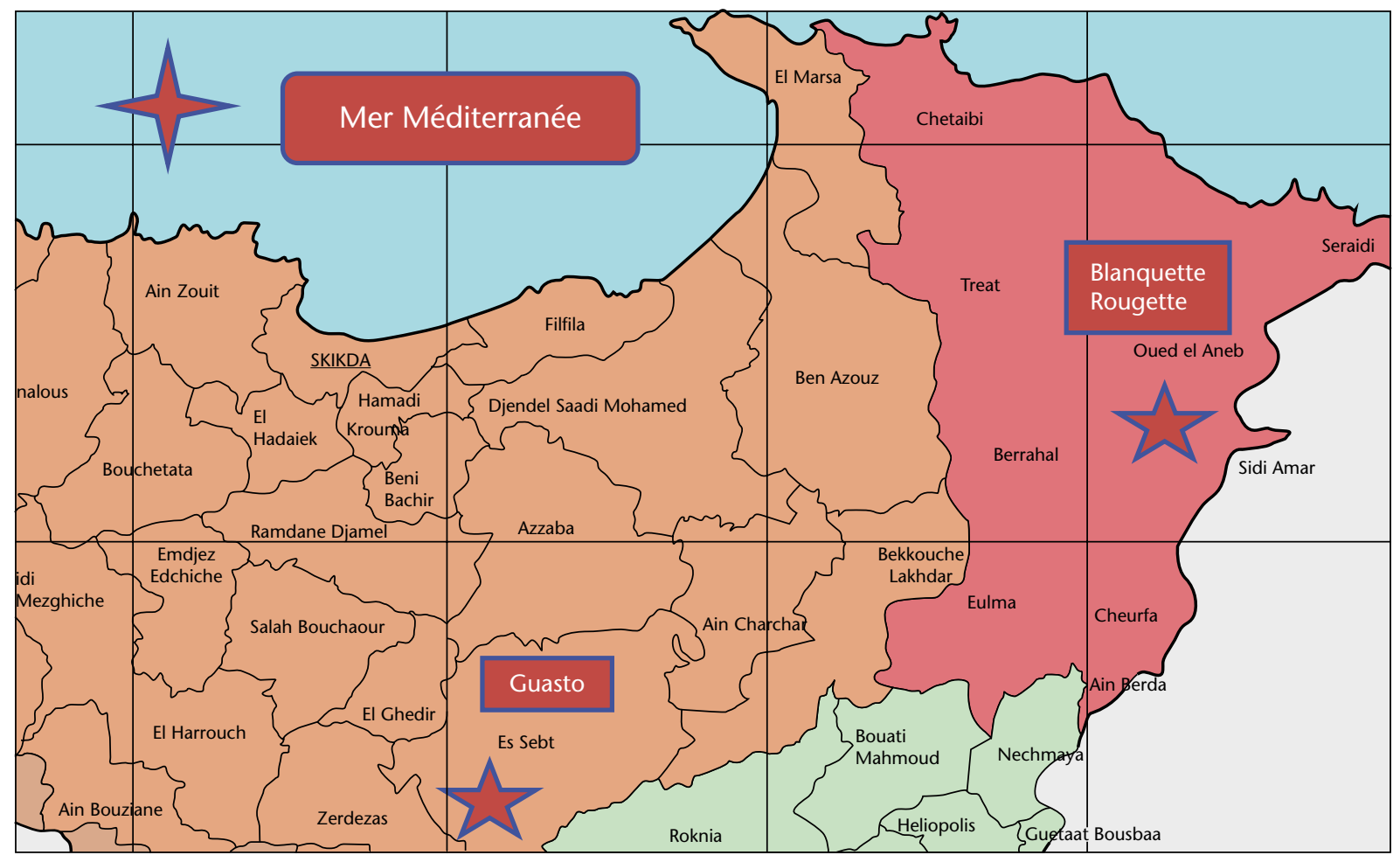

Figure 1. Aires géographiques de production des trois cultivars: Guasto, Blanquette et Rougette. 
Tableau 1. Caractérisation primaire des variétés d'olives (Itaf, 2002).

\begin{tabular}{|lllll|}
\hline Variété & Zone de culture d'origine & Fruit & Utilisation & Arbre \\
\hline Blanquette & $\begin{array}{l}\text { Ain Cherchar, Bekouche lakhdar, } \\
\text { Oued el Aneb (Skikda). }\end{array}$ & $\begin{array}{l}\text { Fruit de bonne fermeté, de faible } \\
\text { poids, de forme allongée, de couleur } \\
\text { rouge violet en pleine maturité, } \\
\text { produit une huile de couleur verte, } \\
\text { fine, fruitée, se conservant bien. }\end{array}$ & $\begin{array}{l}\text { Huile (récolte en } \\
\text { novembre-décembre), } \\
\text { Olive verte (récolte en octobre) }\end{array}$ & $\begin{array}{l}\text { Arbre moyennement vigoureux, } \\
\text { port dressé avec une densité } \\
\text { moyenne du feuillage. }\end{array}$ \\
\hline Guasto & $\begin{array}{l}\text { Es Sebt } \\
\text { (Skikda) }\end{array}$ & $\begin{array}{l}\text { Fruit de poids moyen et de forme } \\
\text { sphérique. }\end{array}$ & $\begin{array}{l}\text { Huile (récolte en } \\
\text { décembre-janvier) } \\
\text { de très bonne qualité. }\end{array}$ & $\begin{array}{l}\text { Arbre de vigueur moyenne, } \\
\text { Port retombant et une densité } \\
\text { compacte du feuillage. }\end{array}$ \\
\hline Rougette & $\begin{array}{l}\text { Bin el ouiden, Kerkra, } \\
\text { Tamalous, } \\
\text { Oued El Aneb (Skikda). }\end{array}$ & Fruit de bonne fermeté, de faible \\
poids, et de forme sphérique. & Huile (récolte en & Aécembre-janvier). & $\begin{array}{l}\text { Arbre de faible vigueur, port } \\
\text { étalé et une densité moyenne } \\
\text { du feuillage. }\end{array}$ \\
\hline
\end{tabular}

ballon (soit un volume final de $80 \mathrm{~mL}$ ). Cette phase est ensuite lavée dans une ampoule à décanter avec $80 \mathrm{~mL}$ d'eau osmosée plusieurs fois jusqu'à l'obtention d'un pH neutre.

L'insaponifiable est évaporé à sec à l'aide de l'évaporateur (type Bushi) puis repris dans $1 \mathrm{~mL}$ du mélange de solvant suivant : hexane (SDS ref. 520048)/Ter-butyle méthyle éther (TBME; Aldrich 23, 321-0) à (90/10, v/v). $100 \mu \mathrm{L}$ du mélange obtenu sont prélevés pour le dosage des phytostérols et autres composants de l'insaponifiable. 3,8 $\mu \mathrm{g} d u$ standard de quantification ( $38 \mu \mathrm{L}$ d'une solution à $0,1 \mathrm{mg} / \mathrm{mL}$ ), le 5alpha cholestane (Sigma, ref C-8003 pur > $97 \%$ ) sont ajoutés dans les $100 \mu \mathrm{L}$.

\section{Silylation de l'insaponifiable extrait [dérivés trimethyl-silyles (TMS)]}

Les dérivés trimethylsilylés (TMS) ont été obtenus selon la méthode décrite par (Park et Addis, 1985). Il s'agit de l'introduction d'un groupement TMS par substitution d'un hydrogène actif. L'action du réactif de silylation, Bis (triméthylsilyl) trifluoroacetamide (BSTFA); (Supelco, ref. 33155-U) est facilitée par la pyridine (Acros, 99,5\%, extra-dry, 339421000 ) et l'addition d'un catalyseur le triméthylchlorosilane (TMCS) ; (Supelco, ref. 33155-U). Le mélange est laissé à $60^{\circ} \mathrm{C}$ pendant 30 min puis évaporé sous azote et $500 \mu \mathrm{L}$ d'hexane sont alors ajouté pour l'analyse en CPG.

\section{Analyse de l'insaponifiable extrait, silylé, par CPG- FID et CPG-SM}

Les TMS sont analysés par CPG sur un chromatographe (Hewlett Packard HP 4890A) équipée d'un détecteur à ionisation de flamme

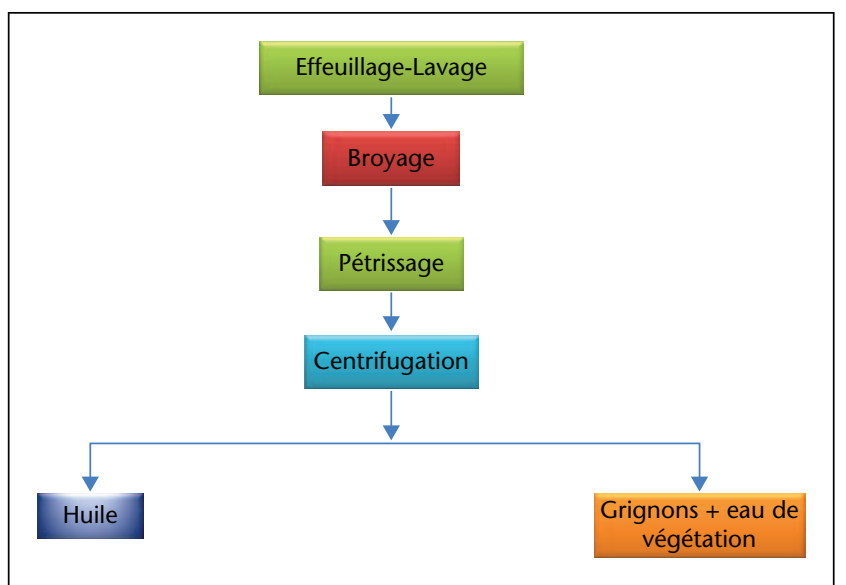

Figure 2. Système continu d'extraction (ALFA LAVAL) avec centrifugation à deux phases.
(CPG-FID) et d'un injecteur de type split/spiltless dont les conditions opératoires sont : colonne RxiTM 5MS (RESTEK) de $30 \mathrm{~m}$ de longueur, $0,25 \mathrm{~mm}$ de diamètre interne et $0,25 \mu \mathrm{m}$ d'épaisseur de film (95\% diméthyle/5\% diphényle polysiloxane); les températures du détecteur et de l'injecteur sont de $300^{\circ} \mathrm{C}$; le gaz vecteur est I'hélium à une pression de 150 Kpa. Système d'acquisition: Galaxie (Varian). Les différents composés ont été quantifiés par rapport au 5alpha cholestane. L'identification des composés a été réalisée par CPG couplée à la spectrométrie de masse (CPG-SM) en utilisant un chromatographe de type AGILENT 6890 A équipé d'un détecteur de masse (MSD 5973N) et un injecteur de type split/spiltless 7673A (Agilent) :

- Colonne DB5 MS chez J and W (122-5532) de 30 m de longueur, $0,25 \mathrm{~mm}$ de diamètre interne et $0,25 \mu \mathrm{m}$ d'épaisseur de film $(95 \%$ diméthyle $/ 5 \%$ diphényle polysiloxane) ; les températures de l'injecteur et de la ligne de transfert sont de $300^{\circ} \mathrm{C}$; le gaz vecteur est I'hélium à une pression de $150 \mathrm{Kpa}$.

- Condition de masse : impacte électronique $(70 \mathrm{eV})$, mode SCAN de 50-500 uma.

- Système d'acquisition : Chemstation (Agilent).

Les différents spectres ont été comparés à ceux de la bibliothèque interne à I'INRA (INRA, 2004).

\section{Analyse statistique}

Les données de nos résultats ont été exprimées par la moyenne de différents essais $(n=3)$ plus ou moins l'écart type et l'analyse statistique a été réalisée au moyen du logiciel Minitab (version 13.31), (X, 2003). Le modèle utilisé est une analyse de la variance (ANOVA) à deux facteurs, modèle fixe.

\section{Résultats et discussion}

\section{Résultats}

\section{Analyse qualitative des composés de l'insaponifiable}

La figure 3 montre le chromatogramme obtenu par CPG-FID de la composition de l'insaponifiable d'une huile d'olive (Guasto 2007). Les sept composés identifiés par CPG-SM dans les huiles étudiées sont : - stérols : sitostérol, delta 5 avenastérol, campestérol et stigmastérol ; - alcools triterpéniques : 24-méthylène cycloartanol et cycloartenol ; - 4-méthyle stérol : citrostadienol.

Les spectres de masse des sept composés ( $A$ à $G$ ) sont présentés dans la figure 4. Ces spectres ont été comparés à une base de données de spectres de masse sur les stérols (INRA, 2004). Cette comparaison a permis l'identification de ces composés. 


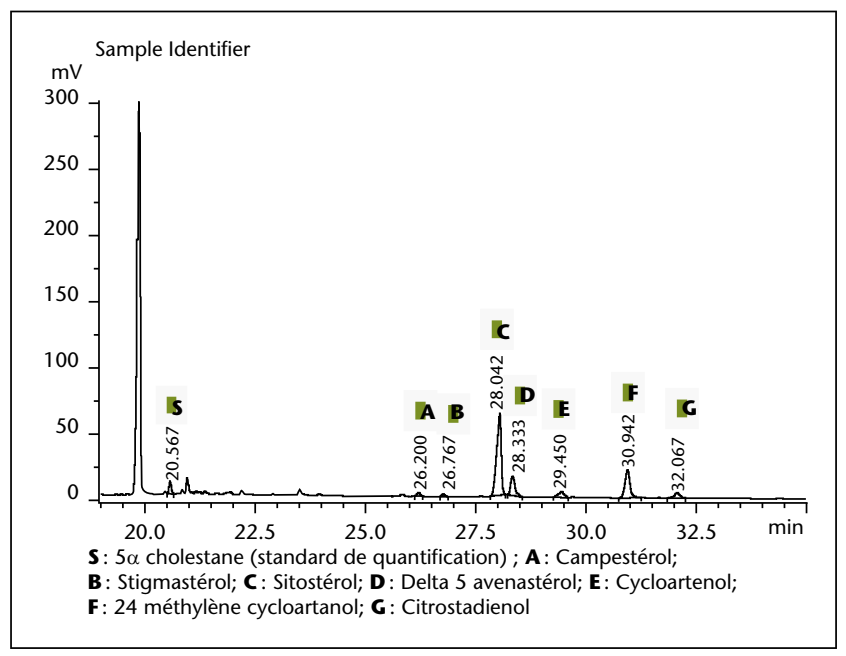

Figure 3. Chromatogramme des différents composés analysés par CPG-FID.

\section{Analyse quantitative}

Le tableau 2 présente les compositions en stérols et composés apparentés exprimées en pourcentage de l'ensemble des composés identifiés dans les différents cultivars Guasto, Blanquette et Rougette, pour trois campagnes successives.

\section{Campagne 2004-2005}

Les résultats présentés dans le tableau 2 montrent que le sitostérol est le stérol dominant suivi par le delta 5 avenastérol et enfin le campestérol et le stigmastérol en plus faible quantité. On remarque également un stérol méthylé, le citrostadienol avec une teneur moyenne de $6 \%$. Des deux alcools triterpéniques, le 24-méthylène cycloartanol est présent en quantité élevée comparé au cycloartenol.

Les teneurs en sitostérol sont plus importantes dans les variétés Rougette et Blanquette $(65,6 \%$ et $66,1 \%$ respectivement) que dans la variété Guasto (43,7\%). Pour le 24-méthylène cycloartanol, le taux le plus élevé est trouvé dans la Guasto (33\%) alors que le plus faible se trouve dans la Blanquette $(11,7 \%)$. Les taux de $\Delta 5$ avenastérol des trois huiles sont très proches. En ce qui concerne les autres composés, tous les échantillons présentent de faibles taux de campestérol: de 1,2\% (Guasto) à 2,1\% (Blanquette); stigmastérol de 0,9\% (Blanquette) à $1,5 \%$ (Guasto); cycloartenol: le taux de la variété Guasto est deux fois plus élevé comparé aux deux autres (6,7 \% pour Guasto, 3,4 \% pour Rougette).

\section{Campagne 2005-2006}

Les résultats montrent que les teneurs en sitostérol sont toujours les plus élevées, allant de 43,2 \% (Rougette) à 62,4\% (Blanquette). On observe cependant de légères baisses par rapport à la campagne précédente. Les teneurs en 24-méthylène cycloartanol, varient de 12,0 \% (Blanquette) à 28,2 \% (Guasto). Enfin les teneurs en $\Delta 5$ avenastérol varient de $9,9 \%$ pour Blanquette à 13,8 \% pour la Rougette. Les deux autres composés les plus importants sont le cycloartenol et le citrostadienol dont les teneurs varient respectivement de 5,4\%(Guasto) à 13,0\% (Rougette) et de 4,9\% (Guasto) à 7,2 \% (Blanquette). Les autres composés stéroliques (campestérol et stigmastérol) sont présents à de faibles taux (inferieurs à $2 \%$ pour les trois cultivars étudiés).

\section{Campagne 2006-2007}

Les résultats présentent toujours une prédominance du sitostérol avec un taux de 55,9\% pour la variété Guasto nettement supérieur comparé aux taux des deux précédentes campagnes, le taux le plus élevé étant encore une fois observé chez la Blanquette $(65,1 \%)$. Le deuxième composé par ordre d'importance est le 24-méthylène cycloartanol avec des taux de $20 \%$ pour Guasto et de 14,2 \% pour Rougette.

Les deux composés cycloartenol et citrostadienol présentent des teneurs assez proches dans les trois variétés (de $4 \%$ à $4,5 \%$ pour le cycloartenol et de 4,1\% à 5,6\% pour le citrostadienol). Enfin, les stérols campestérol et stigmastérol présentent, comme pour les campagnes précédentes, les teneurs les plus faibles $(\leq 2,1 \%)$.

\section{Discussion}

L'ensemble des résultats obtenus au cours des trois campagnes 2004-2005, 2005-2006 et 2006-2007 permet de faire les observations suivantes.

Les résultats obtenus pour les quatre stérols étudiés sont comparables aux spécifications de la norme commerciale du Conseil Oléicole International applicables aux huiles d'olive (COI, 2006); I'Algérie utilise les normes du $\mathrm{COI}$ à défaut de normes nationales.

Les stérols signalés par le COI sont le cholestérol $(\leq 0,5 \%)$, le brassicastérol $(\leq 0,1 \%)$, le campestérol $(\leq 4 \%)$, le stigmastérol (< campestérol pour les huiles comestibles) et le $\Delta 7$-stigmastérol $(\leq 0,5 \%)$. La somme [sitostérol $+\Delta 5$-avenastérol $+\Delta 5,23$-stigmastadiénol + clérostérol + sitostanol $+\Delta 5,24$-stigmastadiénol] doit être égale ou supérieure à $93 \%$ des stérols (ou des méthylstérols) totaux. On observe quatre stérols dans ces huiles : le sitostérol et le $\Delta 5$-avenastérol varient entre 51 et $79 \%$, teneurs inférieures aux spécifications de la norme commerciale du COI ; alors que les quantités de campestérol (taux compris entre 1 et $2 \%$ ) et de stigmastérol (inférieur au campestérol) sont en accord avec les caractéristiques normatives des huiles d'olive. Cependant, la somme des 4 stérols identifiés dans notre étude ne permet pas de conclure qu'elle est comparable à la spécification « $\Sigma$ stérols $\geq 93 \%$ » du COI (COI, 2006).

La présence et les quantités des composés identifiés et dosés présentés dans la figure 3 (profil chromatographique) et la figure 4 (spectres de masse de chaque composé) sont en accord avec d'autres résultats déjà cités dans la littérature (Ben Temime et al., 2008 ; Canâbate-Dıaz et al., 2007 ; Cercaci et al., 2007 ; Pasqualone et Catalano, 2000).

Le plus abondant des phytostérols des trois huiles des cultivars étudiés est le sitostérol, avec un taux de $66 \%$ de l'ensemble des composés quantifiés (Blanquette 2004-2005) ; ces résultats sont en accord avec ceux rapportés pour d'autres variétés d'huiles d'olive (Rivera Del Alamo et al., 2004 ; Ben Temime et al., 2008 ; Pardo et al., 2007). Ils sont parfois comparables aux spécifications du règlement (CEE) $n^{\circ}$ 2568/91 modifié (Annexe I) (CEE, 1991).

Pendant les trois campagnes (figure 5), nous remarquons que le sitostérol est toujours majoritaire, taux plus élevé pour Blanquette et Rougette (Excepté Rougette en 2005-2006) contrairement à la variété Guasto qui présente les taux les plus faibles (43,7\%).

Le 24.méthylène cycloartenol, taux plus élevés dans Guasto et parfois dans Rougette (2005-2006), ce qui semble montrer le caractère particulier de cette campagne; pour le $\Delta 5$ avenastérol, de légères variations ont été observée selon la variété et la campagne, ce taux est légèrement supérieur dans Rougette (2005-2006), pour Guasto et Blanquette les taux les plus élevés ont été observés pendant la campagne 2006-2007. Le cycloartenol : pas de spécificité notable, mais à nouveau particularité de la campagne 2005-2006 pour la variété Rougette (teneur significativement plus élevée). En ce qui concerne le citrostadienol, aucune spécificité (les taux sont assez proches) n'est décelable.

Enfin, les taux du stigmastérol sont toujours inférieurs à ceux du campestérol. En effet, toutes les huiles (excepté pour Guasto de la campagne 2004-2005) répondent à cette spécification (CEE, 1991). 


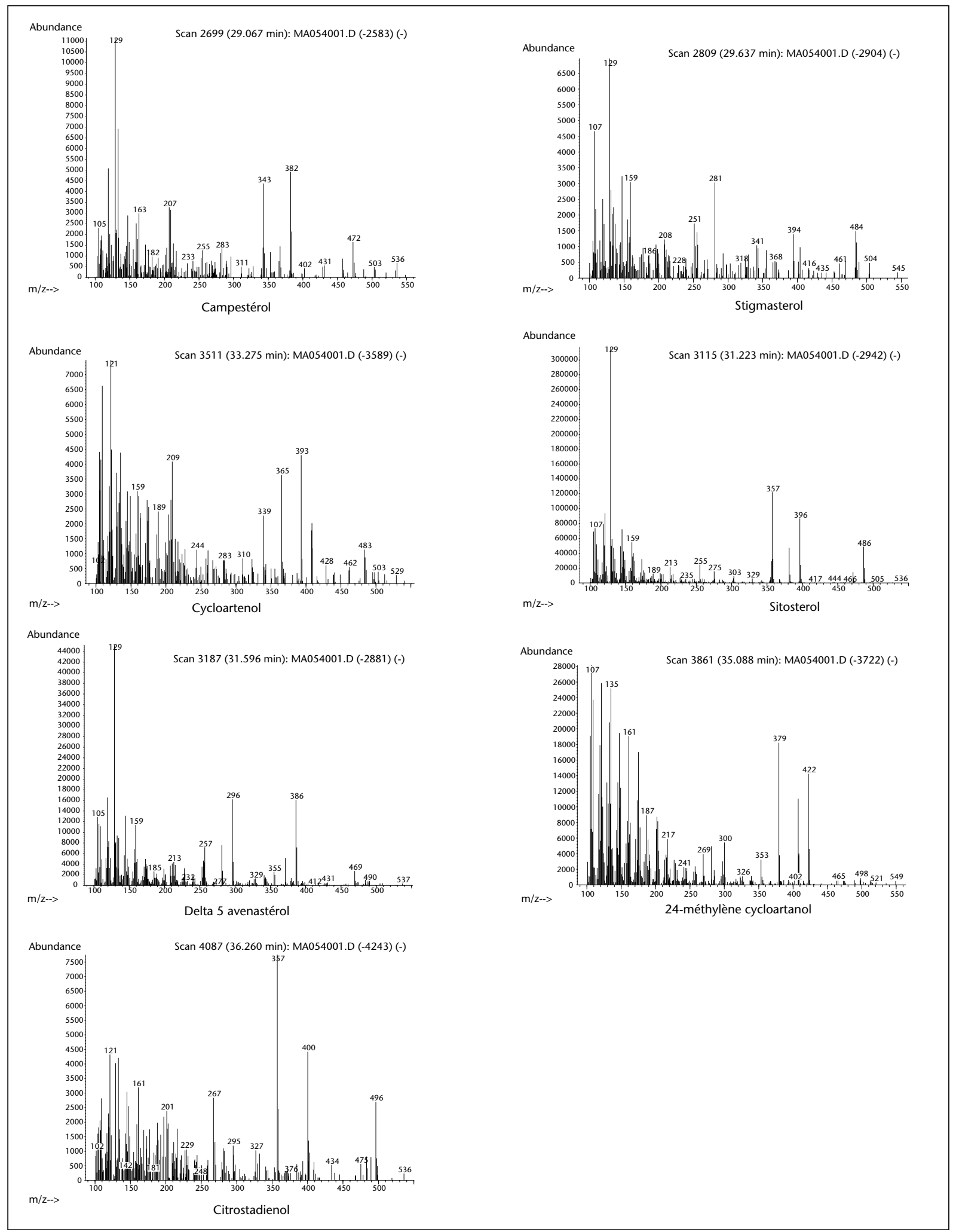

Figure 4. Spectres de masse des sept composés présents dans les huiles d'olive. 
Tableau 2. Analyse des composés de l'insaponifiable (\%) par chromatographie en phase gazeuse (CPG-FID) des trois huiles étudiées pendant les trois campagnes (2004-2005, 2005-2006 et 2006-2007).

\begin{tabular}{|c|c|c|c|}
\hline Composés (\%) & Guasto & Blanquette & Rougette \\
\hline \multicolumn{4}{|c|}{ Campagne (campany) 2004-2005 } \\
\hline \multicolumn{4}{|l|}{ Stérols } \\
\hline Sitostérol & $43,7 \pm 0,66 a$ & $66,1 \pm 0,32 a$ & $65,6 \pm 0,25 a$ \\
\hline Delta 5 avenastérol & $9,5 \pm 0,21 b$ & $9,7 \pm 0,04 b$ & $8,5 \pm 0,10 b$ \\
\hline Campestérol & $1,2 \pm 0,05 c$ & $2,1 \pm 0,05 c$ & $2,0 \pm 0,10 c$ \\
\hline Stigmastérol & $1,5 \pm 0,03 d$ & $0,9 \pm 0,01 d$ & $1,2 \pm 0,01 d$ \\
\hline \multicolumn{4}{|l|}{ Stérol méthylé } \\
\hline Citrostadienol & $4,4 \pm 0,06 \mathrm{e}$ & $5,9 \pm 0,21 e$ & $5,0 \pm 0,39 e$ \\
\hline \multicolumn{4}{|l|}{ Alcools triterpéniques } \\
\hline 24. méthylène cycloartanol & $33,0 \pm 0,58 f$ & $11,7 \pm 0,38 f$ & $14,3 \pm 0,61 f$ \\
\hline Cycloartenol & $6,7 \pm 0,09 \mathrm{~g}$ & $3,6 \pm 0,09 g$ & $3,4 \pm 0,07 \mathrm{~g}$ \\
\hline \multicolumn{4}{|c|}{ Campagne (campany) 2005-2006 } \\
\hline \multicolumn{4}{|l|}{ Stérols } \\
\hline Sitostérol & $47,7 \pm 0,94 a$ & $62,4 \pm 1,10 a$ & $43,2 \pm 0,60 \mathrm{a}$ \\
\hline Delta 5 avenastérol & $11,6 \pm 0,19 b$ & $9,9 \pm 0,51 b$ & $13,8 \pm 0,12 b$ \\
\hline Campestérol & $1,3 \pm 0,18 c$ & $1,9 \pm 0,10 c$ & $1,5 \pm 0,06 c$ \\
\hline Stigmastérol & $0,9 \pm 0,08 d$ & $0,8 \pm 0,05 d$ & $0,2 \pm 0,03 d$ \\
\hline \multicolumn{4}{|l|}{ Stérol méthylé } \\
\hline Citrostadienol & $4,9 \pm 0,34 \mathrm{e}$ & $7,2 \pm 0,34 \mathrm{e}$ & $5,8 \pm 0,23 \mathrm{e}$ \\
\hline \multicolumn{4}{|l|}{ Alcools triterpéniques } \\
\hline 24. méthylène cycloartanol & $28,2 \pm 1,09 f$ & $12,0 \pm 0,48 f$ & $22,4 \pm 0,15 f$ \\
\hline Cycloartenol & $5,4 \pm 0,14 \mathrm{~g}$ & $5,7 \pm 0,79 g$ & $13,0 \pm 0,38 \mathrm{~g}$ \\
\hline \multicolumn{4}{|c|}{ Campagne (campany) 2006-2007 } \\
\hline \multicolumn{4}{|l|}{ Stérols } \\
\hline Sitostérol & $55,9 \pm 0,29 a$ & $65,1 \pm 0,08 a$ & $61,5 \pm 0,63 a$ \\
\hline Delta 5 avenastérol & $12,7 \pm 0,06 b$ & $12,0 \pm 0,09 b$ & $11,0 \pm 0,24 b$ \\
\hline Campestérol & $2,1 \pm 0,09 c$ & $2,0 \pm 0,02 c$ & $2,1 \pm 0,05 c$ \\
\hline Stigmastérol & $1,3 \pm 0,01 d$ & $0,4 \pm 0,02 d$ & $1,1 \pm 0,01 d$ \\
\hline \multicolumn{4}{|l|}{ Stérol méthylé } \\
\hline Citrostadienol & $4,1 \pm 0,20 \mathrm{e}$ & $5,6 \pm 0,03 e$ & $5,5 \pm 0,11 \mathrm{e}$ \\
\hline \multicolumn{4}{|l|}{ Alcools triterpéniques } \\
\hline 24. méthylène cycloartanol & $20,0 \pm 0,14 f$ & $10,7 \pm 0,05 f$ & $14,2 \pm 0,11 f$ \\
\hline Cycloartenol & $4,0 \pm 0,34 \mathrm{~g}$ & $4,2 \pm 0,02 \mathrm{~g}$ & $4,5 \pm 0,37 \mathrm{~g}$ \\
\hline
\end{tabular}

*Résultats exprimés par la moyenne de trois essais \pm l'écart type, $(n=3)$.

Les valeurs désignées par la même lettre (ligne + colonne) sont significativement différentes $(p<0,001)$.

Normes COI : Campesterol $\leq 4.0 \%$; stigmastérol $\leq$ campestérol ; - Somme (Beta-sitostérol + delta-5-avénastérol + delta 5,23-stigmastadiénol + clérostérol + sitostanol + delta 5,24-stigmastadiénol) $\geq 93 \%$. 


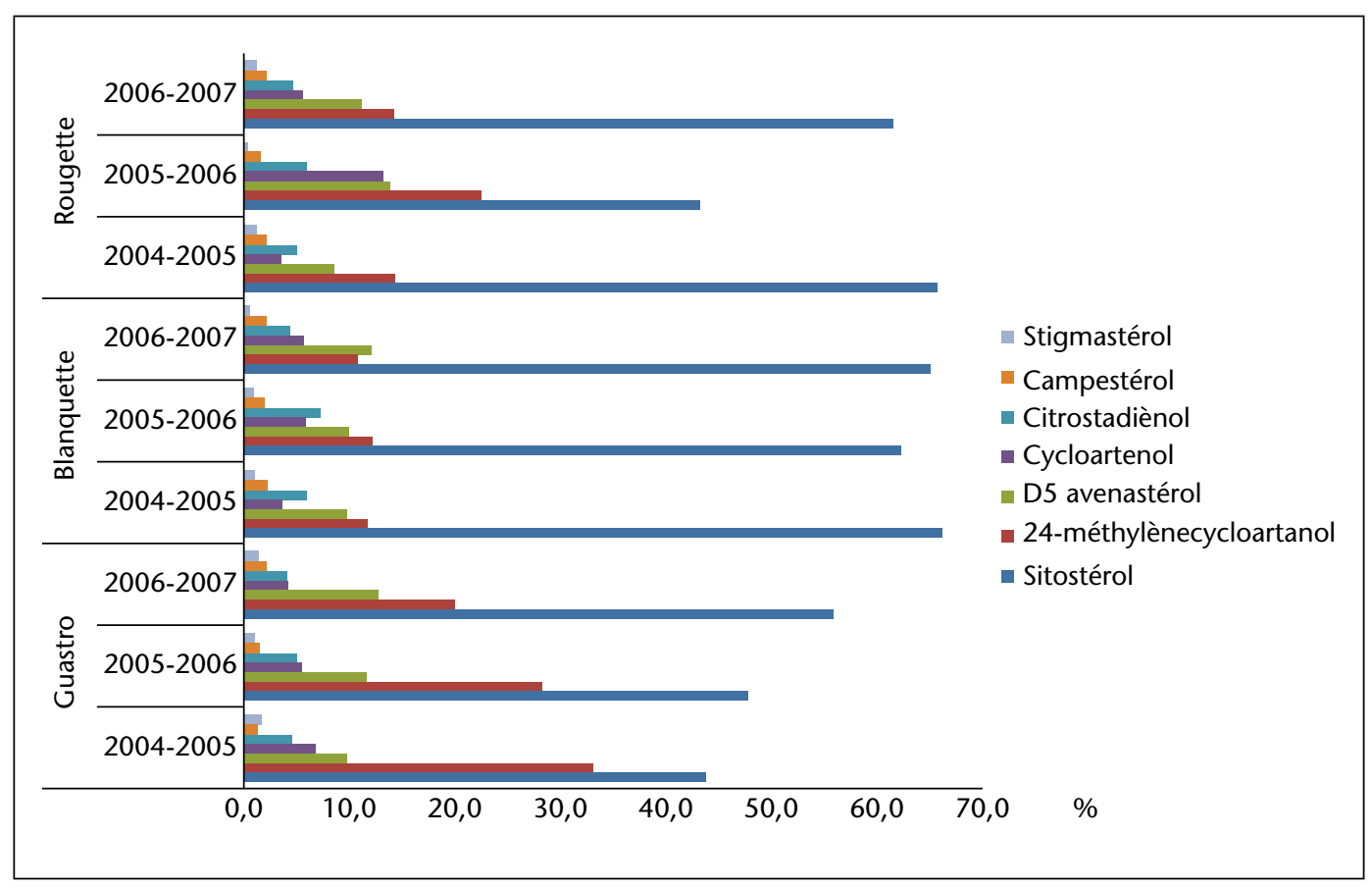

Figure 5. Analyse par chromatographie en phase gazeuse (CPG-FID) des composés de l'insaponifiable (\%) présents dans les trois huiles étudiées (moyennes des trois campagnes).

L'étude statistique a révélé que d'une campagne à l'autre, on observe des différences très hautement significative $(p<0,001)$ pour la variété Guasto et la majorité des stérols et composés apparentés. Les teneurs les plus élevées sont observées pendant la campagne 2006-2007, ce qui pourrait être expliqué par les bilans climatiques (températures et pluviométrie) très favorables pour l'olivier cette année-là (Anonyme, 2007). De même, il existe des différences très hautement significatives $(p<0,001)$ pour certains composés (sitostérol, 24-méthylène cycloartanol) entre les deux variétés d'une même région (Blanquette et Rougette) et la Guasto appartenant à l'autre région, dues probablement à l'existence de microclimats différents, Oued-Aneb étant plus proche de la mer qu'Es-Sebt ; la nature des sols étant aussi très différente (Itaf, 2002). Certains auteurs ont déjà observé des variations dans la composition de l'huile d'un même cultivar en fonction du milieu et des appellations géographiques (Abaza et al., 2002). Cependant, d'autres auteurs (Sanchez et al., 1999) estiment que la variété reste la variable principale qui fait la différence entre les huiles en particulier au niveau de leurs compositions en certains composés mineurs (llyasoglu et al., 2010).

\section{Conclusion}

Les résultats de cette étude ont permis de mettre en évidence et d'identifier par chromatographie en phase gazeuse couplée à la spectrométrie de masse, sept composés de l'insaponifiable d'huiles extraites de trois variétés d'olive et sur trois campagnes successives de production. Ces composés correspondent à quatre stérols (sitostérol, $\Delta 5$ avenastérol, campestérol et stigmastérol), deux alcools triterpéniques (24-méthylène cycloartanol et cycloartenol), et un 4-méthylstérol, le citrostadienol.

Trois composés dominent dans les huiles : le sitostérol, le 24-méthylène cycloartanol et le $\Delta 5$ avenastérol.

Les résultats ont montré des différences du point de vue quantitatif surtout pour les deux composés, sitostérol (taux plus élevés dans Blan- quette et Rougette) et 24-méthylène cycloartanol (taux nettement supérieur dans Guasto et occasionnellement dans Rougette en 2005). Ceci pourrait être expliqué par les changements climatiques observés durant les trois campagnes (une sécheresse, une faible pluviométrie pendant l'hiver et un été très chaud pour les deux campagnes 20042005 et 2005-2006, contrairement aux conditions climatiques très favorables durant l'année 2007) (DSA, 2007).

D'après l'ensemble des résultats obtenus, nous pouvons conclure que les teneurs des différents composés sont parfois dépendantes de la variété (Ollivier et al., 2006 ; Caselli et al., 1993 ; llyasoglu et al., 2010), des conditions climatiques pouvant aussi influencer ces teneurs (Ollivier et al., 2006).

Enfin ce type d'étude pourrait être élargi à d'autres variétés algériennes car il permet d'établir la conformité des huiles d'olive locales aux spécifications du COI et de la réglementation européenne (COI, 2009 ; CE, 2009) et contribue à faire connaître leur valeur sur le marché international (Bedar, 2009 ; Bounekta, 2009).

\section{RÉFÉRENCES}

Abaza L, Msallem M, Daoud D, Zarrouk M. Caractérisation des huiles de sept variétés d'oliviers tunisiennes. OCL $2002 ; 9$ : 174-9.

Anonyme. Rapport sur la situation agricole, direction des statistiques agricoles et des systèmes d'information. Ministère de l'agriculture et du développement rural, Algérie, 2005.

Anonyme. Rapport sur la situation agricole, direction des statistiques agricoles et des systèmes d'information. Ministère de l'agriculture et du développement rural, Algérie, 2006.

Anonyme. Skikda wilaya polyculture, Agriculture et développement. Revue de vulgarisation et de communication 2007; 4.Ed. par l'institut national de la vulgarisation agricole, Algérie. 
Anonyme. Technologie d'extraction de l'huile d'olive et gestion de sa qualité, transfert de technologie en agriculture. Bulletin mensuel d'information et de liaison du PNTTA. Ministère de l'agriculture du développement rural et des pêches maritimes, Royaume du Maroc, 2006.

Bedar L. Huile d'olive. La labellisation en voie de finalisation. Quotidien national, I'Expression, 2009.

Ben Temime S, Manai H, Methenni K, et al. Sterolic composition of Chétoui virgin olive oil: Influence of geographical origin. Food Chem 2008 ; 110 : 368-74.

Bounekta $D$, A la conquête du marché international, I'huile d'olive algérienne va se doter d'un label. Le Maghreb, le quotidien de l'économie, 2009.

Bucci R, Magri AD, Magri AL, Marini D, Marini F. Chemical authentication of extra virgin olive oil varieties by supervised chemometric procedures. J Agri Food Chem $2002 ; 50: 4138$.

Canâbate-Dıaz B, Segura-Carretero A, Fernandez-Gutierrez A, et al. Separation and determination of sterols in olive oil by HPLC-MS. Food Chem 2007; 102 : 593-8.

Caselli S, Modi S, Nizzi Grifi F, Fiorini P. Variabilité de la composition en acides gras, en stérols et en alcools de l'huile d'olive de cultivars de la Toscane. Olivae $1993 ; 47: 46-51$.

CE, Règlement no 182/2009 de la commission du 6 mars 2009 modifiant le règlement (CE) $n^{\circ}$ 1019/2002 relatif aux normes de commercialisation de l'huile d'olive.

CEE, Réglement $n^{\circ}$ 2568/91 modifié, relative aux caractéristiques des huiles d'olive et des huiles de grignons d'olive ainsi qu'aux methods d'analyse y afférentes.

Cercaci L, Passalacqua G, Poerio A, Rodriguez-Estrada MT, Lercker G. Composition of total sterols (4-desmethyl-sterols) in extravirgin olive oils obtained with different extraction technologies and there influence on the oil oxidative stability. Food Chem $2007 ; 102$ : 66-76.

COI (Conseil Oléicole International). Norme commerciale applicable aux huiles d'olive et aux huiles de grignons d'olive. COI/T.15/NC n³/Rév. 4 Novembre 2009.

Di Giovaccino L. L'influence des systèmes d'extraction sur la qualité de l'huile d'olive. Olivae $1996 ; 63: 52-62$

DSA (Direction des services agricoles de Skikda), filière oléicole ; vers une production record. Skikda info. n 009934. Posté le 13 décembre 2007

El Antari A, Hilal A, Boulouha B, El Moudni A. Estudio de la influencia de la variedad, los factores ambientales y la tenicas de cultivo en las caracteristicas de los frutosy la composicion quimicadel aceite de oliva virgen extra de Marruccos. Olivae $2000 ; 80: 29-36$.
Grandgirard A, Martine L, Cordelet C, Juaneda P. Sitostanetriol is not formed in vivo from sitosterol in the rat. Reprod Nut Dev $2004 ; 44$ : 609-16.

Gutierrez F, Varona I, Albi MA. Relation of acidity and sensory quality with sterol content of olive oil from stored fruit. J Agric Food Chem $2000 ; 48$ : 1106-10.

Hajana H. A El Antari A, Hafifi A. Fatty acids, sterol evolution during the ripening of olives from the Moroccan Picholine cultivar. Grasas y Aceite 1998; 49: 405-10.

llyasoglu H, Beraat O, Vera Van H, Roland V. Characterization of Aegean Olive Oils by Their Minor Compounds. J Am Oil Chem Soc 2010 ; 87 : 627-36.

INRA (Institut national de la recherche agronomique). Bibliothèque de spectres. Résultats de I'Unité mixte INRA/ENESAD de Recherche FLAVIC, 21065, Dijon Cedex, France : Flaveur, Vision et Comportement du Consommateur, 2004.

ITAFv (Institut technique de l'arboriculture fruitière et de la vigne). Ministère de l'agriculture et du développement rural, Algérie. Bilan d'activité année 2002.

Jacotot B. Huile d'olive et prévention. Nutr Clin Métabol 1996; 10 : 7S-9S.

Ollivier D. Recherche d'adultération dans les huiles végétales : application à la qualité des huiles vierges et notamment de I'huile d'olive. OCL 2003; 10 : 315-20.

Ollivier D, Artand J, Pinatel C, Durbec JP, Guérère M. Differentiation of french virgin oil RDOCS by sensory characteristic fatty acid and triacylglycerol composition and chemotrics. Food Chem 2006 ; 97 : 382-93.

Pardo JE, Cuesta MA, Alvarruiz A. Evaluation of potential and real quality of virgin olive oil from the designation of origin' Aceite Campo de Montiel (Ciudal Real, Spain). Food Chem 2007 ; 100 : 977-84.

Park SW, Addis PB. Transformation des stérols et des dérivés stérols en dérivés trimethyl-silyles (TMS). Anal Biochem $1985 ; 149$ : 275-83.

Pasqualone A, Catalano M. Free and total sterols in olive oils. Effects of neutralization. Grasas y Aceites $2000 ; 80: 939-66$.

Ranalli A, Pollastri L, Contento S, et al. Sterol and alcohol components of seeds, pulp, and whole olive fruit oils. Their use to characterize olive fruit variety by multivariate. / Sci Food Agric $2002 ; 82: 854-9$.

Rivera Del Alamo RM, Fregapane G, Aranda F, Gomez-Alonso S, Salvador MD. Sterol and alcohol composition of Cornicabra virgin olive oil : The campesterol content exceeds the upper limit of $4 \%$ established by EU regulations. Food Chem $2004 ; 84$ : 533-7.

Sanchez Casas IJ, De Miguel Gordillo C, Marin Exposito J. La qualité de I'huile d'olive provenant de variétés en Estrémadure en fonction de la composition et la maturation de l'olive. Olivae $1999 ; 75$ : 31-6.

X, 2003. Minitab version (13.31). Statistical analysis of Microsoft Software. 